\title{
IMPACT OF PERIODONTITIS ON THE ECG DISPERSION MAPPING OF THE MYOCARDIAL TISSUES
}

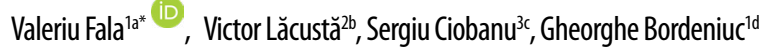 \\ 'Department of Therapeutic Dentistry, Faculty of Dentistry, “Nicolae Testemițanu” State University of Medicine and Pharmacy, Chișinău, Republic of Moldova \\ 2Department of Alternative and Complementary Medicine, Faculty of Medicine, “Nicolae Testemițanu”S State University of Medicine and Pharmacy, Chișinău, \\ Republic of Moldova \\ 'Department of Odontology, Periodontology and Oral Pathology, Faculty of Dentistry, "Nicolae Testemițanu” State University of Medicine and Pharmacy, \\ Chișinău, Republic of Moldova
}

${ }^{a} D M D$, PhD, MSc, Assistant Professor, Head of the Department of Therapeutic Dentistry

${ }^{\mathrm{b}} \mathrm{DMD}, \mathrm{PhD}$, Academician, Professor

'DMD, PhD, Professor, Dean of the Faculty of Stomatology

${ }^{d} D M D$, University Assistant

\section{ABSTRACT \\ DOI: https://doi.org/10.25241/stomaeduj.2019.6(3).art.1}

Introduction: The periodontal disease is considered to have an epidemic reach in the general population. The impact of the periodontal disease on the general health is studied from the perspective of the existence of complex interrelationships, especially with respect to the cardiovascular system. The aim of the study was the assessment of the correlation between the clinical signs of the chronic periodontitis and the changes in the ECG dispersion of the myocardial tissues and the analysis of the efficiency of the masticatory test and full mouth disinfection procedure for recording these signs in patients with chronic periodontitis.

Methodology: 92 patients with chronic periodontitis without the clinical manifestations of the cardiovascular disease and pathological signs on standard ECG were included in the study. Periodontitis was diagnosed based on the clinical and radiological exams.The ECG dispersion mapping technology was used to identify changes in the myocardial tissues, under various conditions.

Results: The pathological system of periodontitis-target organ (heart), its elements and the pathways of interaction were described. The research outlined the diagnostic value of the masticatory test and of the full mouth disinfection procedure in the process of identifying the changes in ECG dispersion of the myocardial tissues in patients with chronic periodontitis. It also proposed an algorithm for the interdisciplinary management of patients with chronic periodontitis, based upon the periodontitis-target organ (heart) concept.

Conclusion: The periodontitis-target organ (heart) concept represents a cumulative synthesis of the available data from literature and of our investigations, and it may prove as a step towards an integrative medicine approach in periodontology.

Keywords: Interdisciplinary management; Index myocardium; Periodontal index; Periodontics; Target organ.

\section{Introduction}

According to the epidemiological studies published by $\mathrm{WHO}$, gingivitis and periodontitis can be classified as presenting epidemic traits, even in highly developed countries - severe chronic periodontitis affects about $35 \%$ of the US population and it is forecast that by 2030 , the number of individuals with chronic periodontitis in the US will reach 71 million (1 in 5 individuals) $[1,2]$. Chronic periodontitis is a public health issue, not only based on the dramatic increase in the number of patients, but also due to serious medical, social and economic consequences. Currently, the relationship between the periodontal disease and general health is being thoroughly studied $[3,4]$. The notion of periodontal medicine has

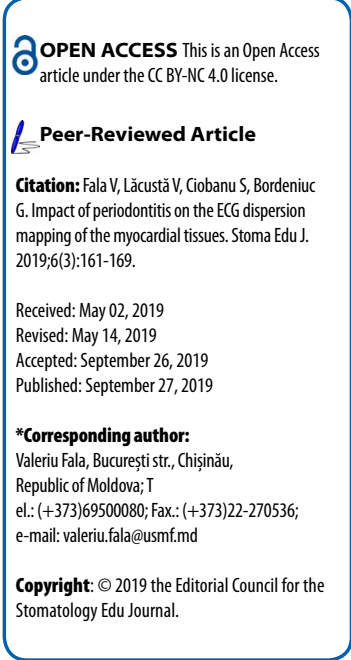

emerged, which refers to the multiple and complex interrelationship between periodontal diseases and general health. In 1891, W. D. Miller described the oral cavity as the infection foci, by which bacteria and their products enter adjacent or remote parts of the body. There are three ways of interaction between the affected periodontium and non-oral organs: bacteremia, systemic inflammation (interleukins, etc.) and endotoxemia caused by swallowed bacteria [5].

Hypothesis: For patients with chronic periodontitis, ischemic myocardial disorders develop gradually, and at the initial stages they may be asymptomatic afterwards they intensify with the increase in the disease's duration and severity. 


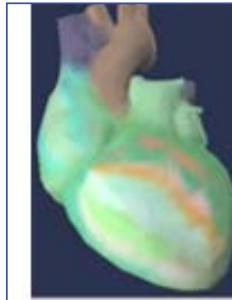

A

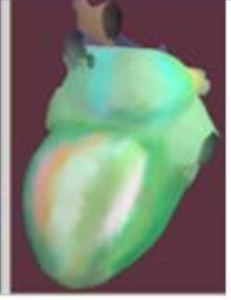

$\mathrm{IM}=4 \%$

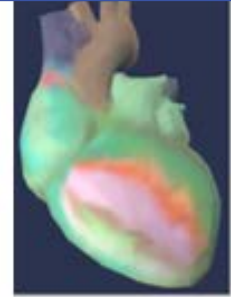

B

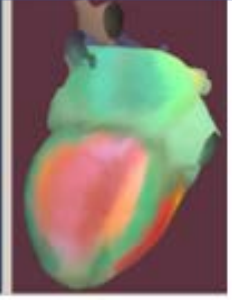

$\mathrm{IM}=59 \%$
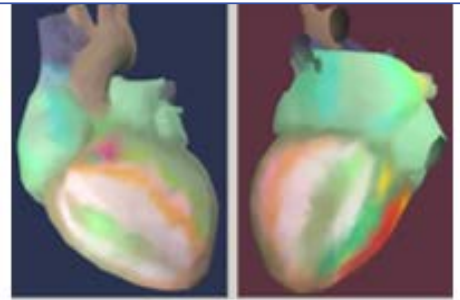

$\mathrm{C}$

$\mathrm{IM}=27 \%$

Figure 1. Values of the Index Myocardium before and after the masticatory test on a patient with chronic periodontitis (48yr. old). A - before the masticatory test, IM has a normal value $(\mathrm{IM}<15)$;

B-20 minutes after the masticatory test, IM has a pathological value $(I M>15)$;

C-60 minutes after the masticatory test, IM has a pathological value $(\mathrm{IM}>15)$.

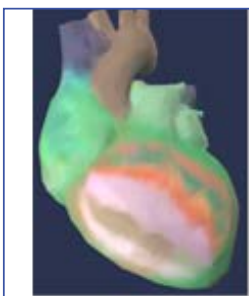

A

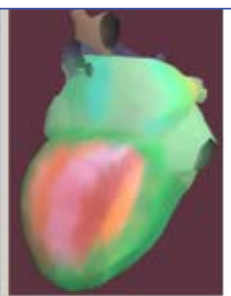

$\mathrm{IM}=36 \%$

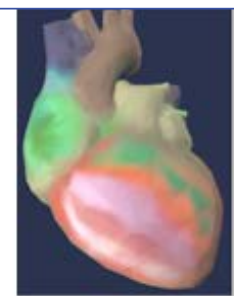

B

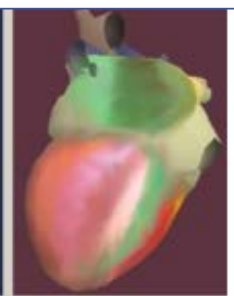

$\mathrm{IM}=62 \%$
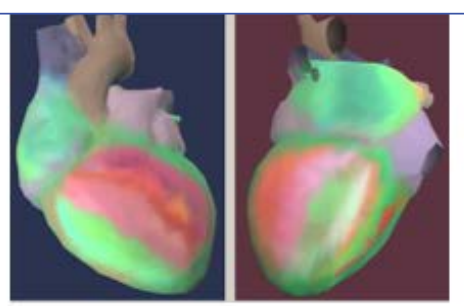

C

$\mathrm{IM}=40 \%$

Figure 2. Values of the Index Myocardium before and after the masticatory test on a patient with chronic periodontitis ( $52 \mathrm{yr}$. old).

A- before the masticatory test, IM has a pathological value (IM > 15);

$B-20$ minutes after the masticatory test, IM has a pathological value $(I M>15)$;

$\mathrm{C}-60$ minutes after the masticatory test, IM has a pathological value $(\mathrm{IM}>15)$.

Aim of the study: Assessment of the correlation between the clinical signs of the chronic periodontitis and the changes in ECG dispersion of the myocardial tissues and analysis of the efficiency of the masticatory test and full mouth disinfection procedure for recording these signs in chronic periodontitis.

\section{Materials and Methods}

92 patients with chronic periodontitis (aged between $25-58$ years) without clinical manifestations of cardiovascular disease and pathological signs on standard ECG analysis (after a prior exam at the cardiologist) were selected for this study. Periodontitis was diagnosed following the clinical and radiological exam. The ECG dispersion mapping $[1,6]$ was used to identify changes in the ECG dispersion of the myocardial tissues, under various conditions (relaxed state, mastication test - during 2 minutes, full mouth disinfection procedure (FMDP) in 2 stages/24h).

\subsection{ECG dispersion mapping}

Currently, electrocardiography (ECG) is one of the most widespread medical methods, with wide application not only in cardiology and although there is still an ongoing development of standard ECG methods, they still have a low specificity and sensitivity (30-40\%) to identify myocardial ischemia [7]. This means that in $60 \%$ of cases, the clinical forms of myocardial ischemia will remain undiagnosed.

The ECG dispersion mapping technology is based on the analysis and visualization of the electromagnetic radiation of the myocardium, with the recording of the small amplitudes fluctuations. The quantitative and qualitative characteristics of the amplitude fluctuations were analyzed using the CardioVisor06s device (Medical Computer Systems, Russian Federation). The technology allows recording multiple indices, including the Index Myocardium (IM), which quantitatively reflects the myocardial ischemic processes in a range between $0-100 \%$. In healthy individuals, the Index Myocardium does not exceed $15 \%$. A higher value of the index translates into more pronounced myocardial ischemic disorders. It is to be noted that the ECG dispersion mapping technology is much more informative compared to the standard ECG myocardial ischemic disturbances can be detected in individuals who show normal values of the standard ECG indices. The diagnostic value of this method for identifying myocardial ischemic disturbances is very high - sensitivity $>80 \%$ [7].

\section{Results}

The ECG dispersion mapping makes it possible to identify not only the organic, but also the functional disturbances of the myocardium. This has provided the opportunity to highlight ischemic disorders at a preclinical level. We have determined that in $85 \%$ cases of patients with chronic periodontitis, preclinical ischemic myocardial disorders were present (Index Myocardium > 15). 


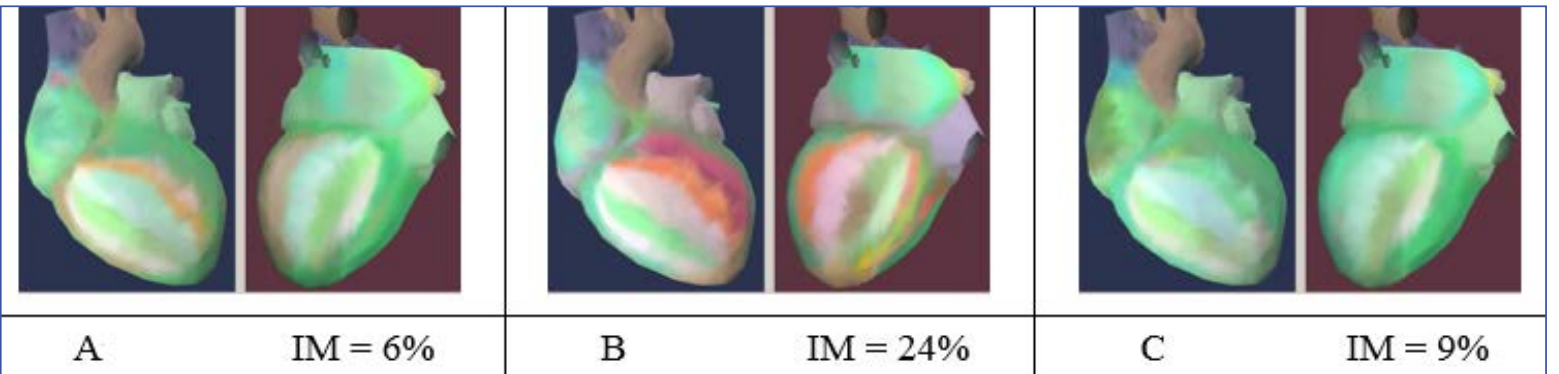

Figure 3. Values of the Index Myocardium (normal initial IM value) before and after the full mouth disinfection procedure (1st phase - mandible). A- before FMDP $(\mathrm{IM}<15)$;

B-20 minutes after FMDP $(\mathrm{IM}>15)$;

C - $12 \mathrm{~h}$ after FMDP $(\mathrm{IM}<15)$.

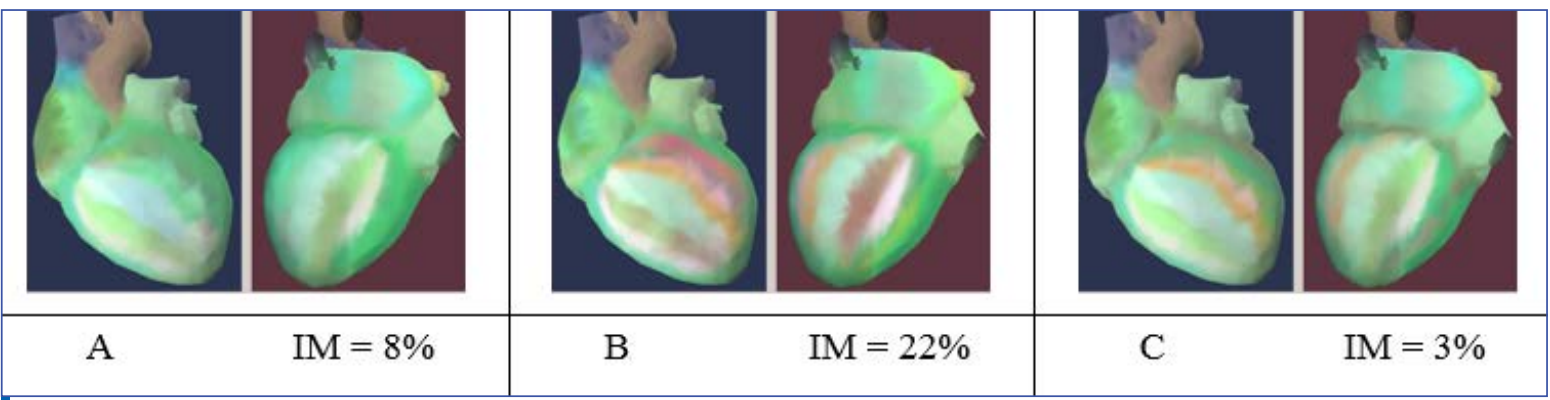

Figure 4. Values of the Index Myocardium (normal initial IM value) before and after the full mouth disinfection procedure (2nd phase-maxilla).

A- before FMDP $(\mathrm{IM}<15)$;

B- 20 minutes after FMDP $(\mathrm{IM}>15)$;

C - $12 \mathrm{~h}$ after FMDP $(\mathrm{IM}<15)$.

We have studied the correlation between the Index Myocardium (IM) and the Integrative Periodontal Index (IPI - synthesis of common periodontal indices - PMA, OHI-S, PI, PBI, TM), disease duration, patient's age and systolic blood pressure (SBP).

The correlation analysis has shown that with the increase in the severity of the periodontitis, the value of the Index Myocardium (ischemic signs) will be higher; the duration of the chronic periodontitis has a stronger correlation with the Index Myocardium $\left(r_{x y}=0.59, p<0.01\right)$ in comparison to the age factor $\left(r_{x y}=0.42, p<0.05\right)$, which demonstrates that the duration of pathological periodontal process has a more negative impact on the ischemic myocardial disorders. Although in this study, patients without pathological values of SBP were included, statistically significant correlations were observed between SBP and the Index Myocardium $\left(r_{x y}=0.46, p<0.05\right)$. This demonstrates that the SBP variations, even if they are within the normal range, have a significant influence upon the ischemic myocardial disorders - with the increase of the SBP values, the preclinical ischemic myocardial signs are more expressed.

3.1. ECG dispersion mapping dynamics under the influence of the masticatory test on patients with chronic periodontitis

The analysis of data from patients with chronic periodontitis and initial normal values of the Index Myocardium $(\mathrm{IM}<15)$ has shown the following changes in comparison with the values observed in a relaxed state (48 patients/100\%):

- for 10 patients (20.8\%), the Index Myocardium has increased 20 minutes after conducting the masticatory test;

- $\quad$ for 38 patients (79.2\%), the Index Myocardium has deviated $\sim 1-2 \%$ (without significant changes).

The analysis of the data from patients with chronic periodontitis with initial pathologic values of the Index Myocardium (IM >15), has shown the following changes in comparison to the values observed in a relaxed state (46 patients/100\%):

- for 17 patients (37\%), the Index Myocardium has increased 20 minutes after the masticatory test;

- for 29 patients (63\%) the Index Myocardium has deviated $\sim 1-2 \%$ (without significant changes).

3.2. ECG dispersion mapping dynamics under the influence of the full mouth disinfection procedure (FMDP) on patients with chronic periodontitis

Preclinical ischemic changes in the myocardium (IM index) in patients with chronic periodontitis under the influence of the full mouth disinfection procedure appear in 2 variants - monophasic or biphasic. The first phase - IM-aggravation phase (worsening of the preclinical ischemic signs) starts within the first minutes and hours after the full mouth disinfection procedure; and is probably caused by transitory bacteremia. The second phase - IM-reduction phase (mitigation of preclinical ischemic signs) starts after several more hours, after the full mouth disinfection procedure, possibly due to the elimination of the periodontal foci, with a reduction in the degree of bacterial invasion 


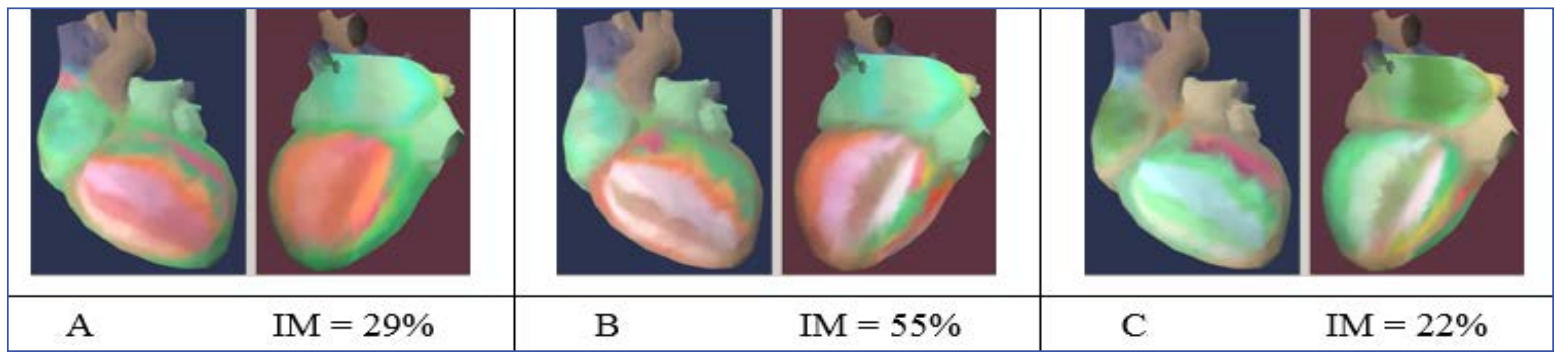

Figure 5. Values of the Index Myocardium (pathological initial IM value) before and after the full mouth disinfection procedure (1st phase - mandible). A- before FMDP $(I M>15)$;

B-20 minutes after FMDP $(\mathrm{IM}>15)$;

C - $12 \mathrm{~h}$ after FMDP $(\mathrm{IM}>15)$.

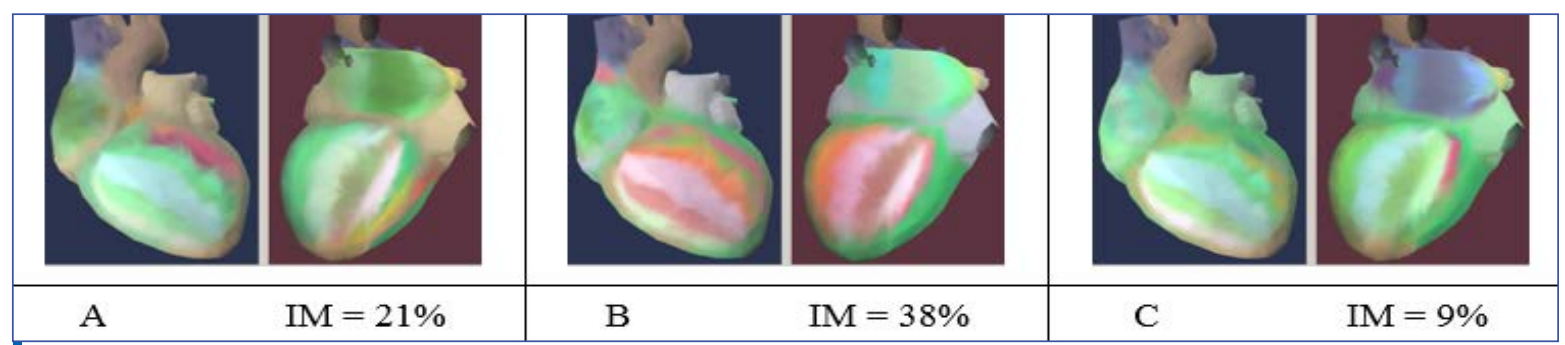

Figure 6. Values of the Index Myocardium (pathological initial IM value) before and after the full mouth disinfection procedure (2nd phase - maxilla). A- before FMDP $(\mathrm{IM}>15)$;

B-20 minutes after FMDP $(\mathrm{IM}>15)$;

C - $12 \mathrm{~h}$ after FMDP $(\mathrm{IM}<15)$.

and dissemination into the bloodstream. In $79.8 \%$ cases, during FMDP, both phases can be observed (a biphasic response - aggravation/reduction). In $20.2 \%$ cases, the aggravation phase is maintained for a longer time, without transitioning to the reduction phase (monophasic response). It is to be noted that when the values of the Index Myocardium are higher than $50 \%$ or if FMDP induces only a monophasic response, it is advised to conduct the consequent dental treatment under the surveillance of a cardiologist.

\section{Discussion}

Numerous studies have been published in the last decade, that indicate a close relationship between periodontal disease and systemic diseases (cardiovascular diseases, diabetus mellitus, lowpreterm birth-weight, osteoporosis, etc.), especially highlighting the more profound impact in regard to the cardiovascular system. The analysis of the data from the literature and of our own results enabled us to identify a pathological system - the periodontitistarget organ (heart) (Fig. 7).

Other organs may serve as target organs for periodontitis (liver, lungs, pancreas, kidneys, etc.). Currently, most data, however, are related to the relationship between chronic periodontitis and heart disease $[3,8,9,10]$, and may be summarized as follows:

- the presence of common risk factors for chronic periodontitis and cardiovascular diseases (smoking, stress, etc.);

- frequent association of chronic periodontitis with various cardiac conditions (stenocardia, myocarditis, atherosclerosis, etc.);

- the presence of certain bacterial agents (T. forsynthensis, T. denticola, P. gingivalis, A. actinomycetemcomitans), simultaneously in the periodontal tissues and in the endothelium of the cardiac vessels;

- experimental administration of Porphyromonas gingivalis in animals leads to the occurrence of myocarditis and myocardial infarction and induces a pro-coagulant response to the vasculopathic effects; a simultaneous worsening of the inflammation degree of the periodontium and myocardium, at the injection of certain proinflammatory factors;

- bacteremia in patients with chronic periodontitis leads to the thickening of the heart valves;

- acceleration of atherogenic processes due to periodontal pathogens; followed by the destabilization of atherosclerotic plaques under the influence of periodontal bacteria that enter the endothelium and the vascular myocytes; exacerbation of inflammation with a progression of the atherosclerotic plaque, based on immune mechanisms, under the influence of periodontal bacterial products (molecular mimicry);

- the existence of common toxic factors in chronic periodontitis and cardiovascular diseases;

- the existence of common mechanisms in the process of diminishing dento-periodontal support and bone resorption, with the occurrence of cardiovascular disturbances - each $20 \%$ bone loss is associated with an increase in 


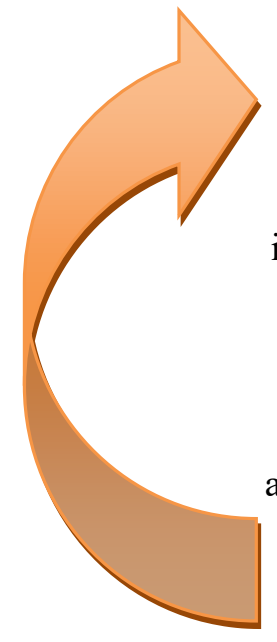

susceptibility to periodontal disease, effects of risk factors, poor oral hygiene

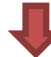

reduction of the body's defense capabilities and of the anatomical-physiological barriers in the stomatognathic

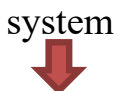

primary infection of the oral cavity and of the dentoperiodontal structures

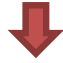

critical noxious burden (bacteria/pathogens), penetration of the defence (barriers), initiation of inflammatory processes in the periodontium

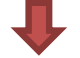

chronicization of the pathological process, progressive damage to the dento-periodontal structures, creation of a continuously-renewing pathogenic periodontal reservoir

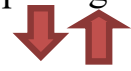

invasion of bacteria/pathogens from the periodontal reservoir into the systemic bloodstream

(bacteremia/toxemia/endotoxemia, etc.), the onset and development of systemic disorders

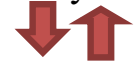

adherence of bacteria to various non-oral structures and target organs (locus minoris resistentiae), their colonization, formation of multidirectional connections, establishment of the periodontitis-target organ pathological system

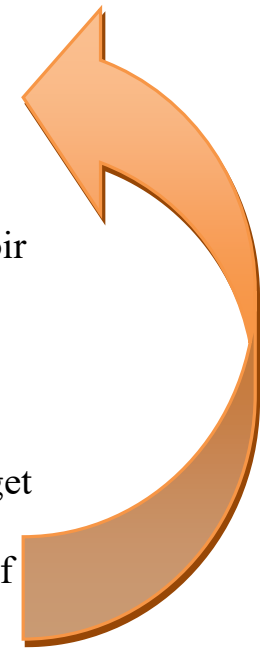

Figure 7. The periodontitis-targetorgan (heart) pathological system.

the incidence of cardiovascular diseases by $40 \%$; chronic periodontitis and tooth loss increases the risk of fatal events in cardiac patients;

- diminishing the severity of the pathological manifestations of chronic periodontitis under the influence of treatment, simultaneously with the reduction of the morpho-functional disorders in the cardiovascular system;

- existence of a common genetic predisposition for periodontal and heart disease; the genes for the immune and inflammatory response inter-penetrate and influence the expression of a hyper-inflammatory phenotype, that predisposes both to coronary atherosclerosis and to periodontal disease.

Our own investigations revealed the presence of preclinical ischemic myocardial disturbances (PIMD), evidenced by changes in ECG dispersion of the myocardial tissues in patients with chronic periodontitis, which demonstrates the role of the heart as a target organ for periodontitis at a preclinical level: the frequent occurrence of PIMD in patients with chronic periodontitis (85\% of cases); diminution/disappearance of PIMD after efficient treatment of chronic periodontitis; reduction/ disappearance of PIMD after the removal of occlusal trauma in patients with chronic periodontitis; aggravation of PIMD in patients with chronic periodontitis under the influence of the masticatory test and the professional oral hygiene procedure (microtrauma, transient bacteremia); the positive correlation between the severity of the chronic periodontitis and the duration of the disease with the PIMD expression.

The analysis of the results obtained in combination with the data from the literature allowed us to highlight the main ways of interaction in the periodontitis-target organ (heart) pathological system: interaction based on the infectious factor (bacteremia); neurogenic interaction; humoralmetabolic interaction, immune and toxic factors; interaction based on genetic determinism.

The bacterial factor is one of the most important in the periodontitis-target organ (heart) system.

Actinobacillus actinomycetemcomitans, Campylobacter rectus and Eikenella corrodens are most commonly found in periodontal processes, but under various pathological conditions, the role of major bacteria can be played by various species. In patients with chronic periodontitis, more than 700 


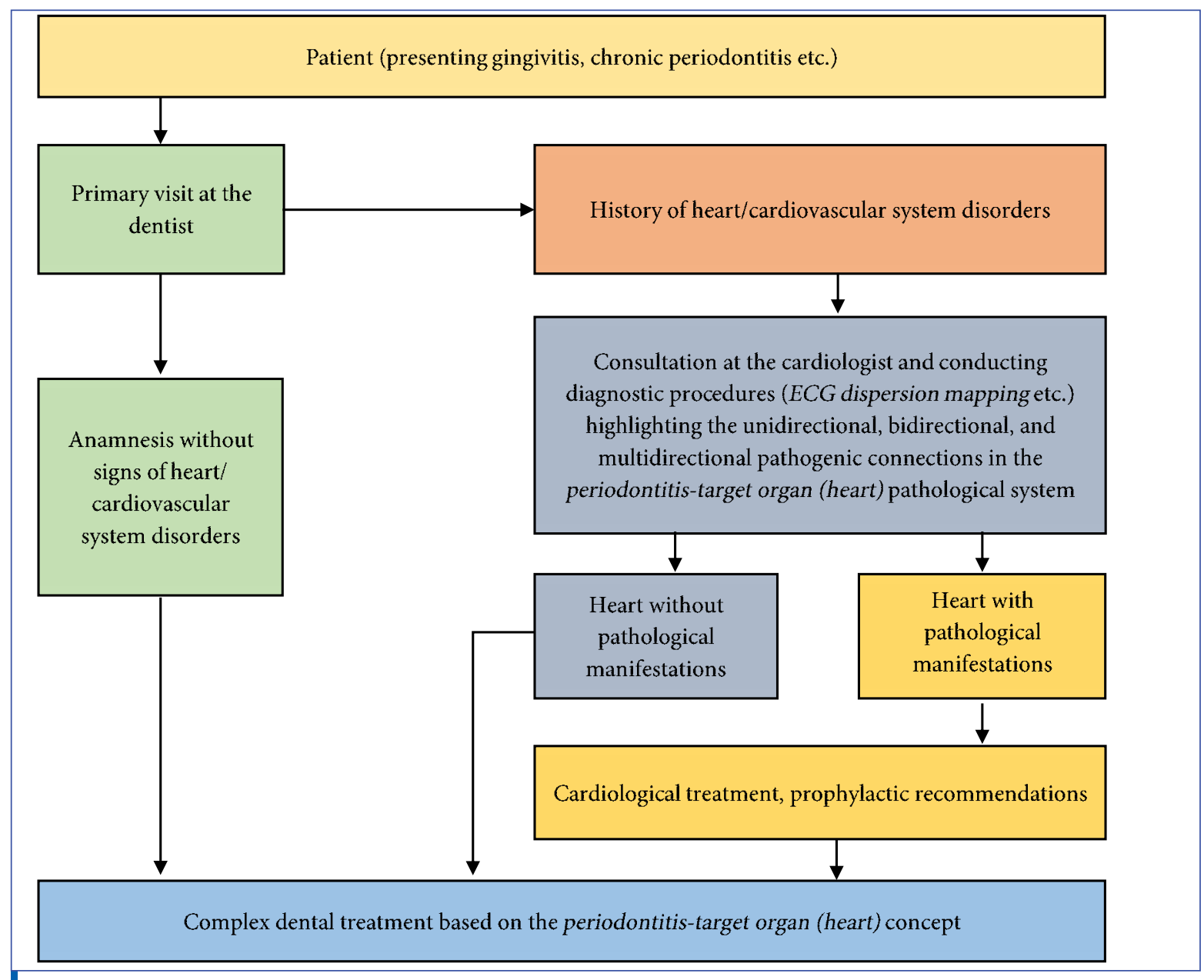

Figure 8. Algorithm of the interdisciplinary management of patients with chronic periodontitis (in the vision of the periodontitis-target organ (heart) concept).

bacterial species have already been detected [11]. Periodontal bacteria can induce proliferation of smooth muscle cells in the vessels of the heart, causing destruction and endothelial damage, vasomotor functional disorders [7]. In our studies, we highlighted the distinct phases of the changes in ECG dispersion of the myocardial tissues, under the effect of the FMDP, associated with the occurrence of transient bacteremia: the worsening phase (the first minutes and hours, during and after the procedure) and the mitigation phase of the ischemic disturbance (hours and days after the procedure).

In $79.8 \%$ cases, both phases (worsening/mitigation) are manifested, and in $20.2 \%$ cases the worsening stage persists without a transition to the mitigation phase, which is an indication for additional investigations/monitoring by the cardiologist. Therefore, the dentist, based on the peculiarities of the chronic periodontitis management, may be the first specialist to detect the presence of preclinical ischemic myocardial disorders associated with chronic periodontitis, based on the detection of changes in the ECG dispersion of the myocardial tissues.

Another point is represented by the neurogenic interaction, which is fundamental in achieving sanogenic and pathogenic connections, which are present in the case of chronic periodontitis. The afferent part of the trigeminal-cardiac reflex consists of the somatosensory trigeminal terminations, the Gasser node, and the trigeminal sensory nuclei. Afterwards, via interconnecting short internuclear fibers, there is a link established with the structures of the reticulated formation and with the motor nuclei of the vagus nerve.

Various pathological irritations, dental manipulations in the area innervated by the trigeminal nerve can cause the trigeminal-cardiac reflex [12]. In recent years, the maxillo-mandibulo-cardiac reflex has been described, with pronounced bradycardic effects. Arakeri et al. describes the dento-cardiac reflex, which is manifested at the irritation of the maxillary jaw (hypotension, syncope, hyperhidrosis, bradycardia) [13]. This reflex may be observed in patients without cardiovascular disease.

The interaction of humoral-metabolic, immune and toxic factors is another important component in the periodontitis-target organ (heart) pathological system. There is a strong interaction between periodontal metabolic disorders and the cardiovascular pathology - IL-1 $\beta$, IL-6, TNF- $\alpha$, PGE2 and other pro-inflammatory factors produced in the affected periodontal tissues, may enter the bloodstream and casue various infections at remote 
sites, including in the heart $[3,14]$.

A series of endocrine-like substances [15] are produced in the periodontal foci, with immune complexes being formed, that amplify the inflammation, both in the dento-periodontal region and in the tissues of the heart $[10,20]$.

The affected marginal periodontium represents a continuously-renewing reservoir with a permanent secretion of toxic substances into the bloodstream, inducing and perpetuating the pathological effects and systemic disorders of the internal organs, including the heart.

In patient with periodontitis, even mild mastication already releases bacterial endotoxins from the oral cavity into the bloodstream with harmful effects on the cardiovascular system.

Applying ECG dispersion mapping as a screening test for the preclinical myocardial ischemic disorders by detecting changesinECG dispersion of the myocardial tissues, in patients with chronic periodontitis has allowed us to highlight the diagnostic value of this method: Se (sensitivity) - 36.9\%, Sp (specificity) - $79.1 \%$, +PV (positive predictive value) - 62.9\%, -PV (negative predictive value) - $43.2 \%$. These data demonstrate that the masticatory test and the ECG dispersion mapping can be applied first of all, in order to exclude ischemic processes involving the myocardium in patients with periodontitis.

Genetic factors are studied insufficiently, although the DNA of the microbiota represents $99 \%$ of the collective genome of the human body [16]. The first to demonstrate the association between periodontitis and genetic determinism was Michalowicz et al. [17]. In heterozygous and homozygous mice with a congenital apolipoprotein-E deficit, there was a high risk of atherosclerosis of the aortic and cardiac vessels at the intravenous administration of $P$. gingivalis [6]. A relationship between a specific polymorphism of the IL-1 genotype and the phenotypic expression of the marginal periodontitis was detected.

The increased risk for severe periodontitis in individuals with a positive genotype (periodontitis susceptibility test) is estimated to be 6.8 times higher compared to subjects with a negative genotype. It is estimated that about $30 \%$ of the population may be positive for this genetic marker [4]. There is a common genetic predisposition for periodontitis and heart disease [18].

Highlighting these interactions based on the periodontitis-target organ (heart) concept - provides the opportunity to monitor the patient's condition and to optimize the complex personalized treatment programs in accordance with an interdisciplinary algorithm for the management of patients with chronic periodontitis (Fig. 8).

The first results in regard to the optimization of the diagnosis and complex treatment based on the proposed concept seem to be promising. Thus, in order to act on the interaction pathways in the periodontitis-target organ (heart) system, we applied biologically active preparations (BioR ${ }^{\circ}$ ), the pituitary neuropeptide - oxytocin, transcranial directcurrent stimulation, transcutaneous electric nerve stimulation (TENS) $[8,19,20]$.

Based on the periodontitis-target organ (heart) concept, it is recommended to monitor the risk factors of cardiovascular disorders during dental treatment; the dental consultation should include gathering information on cardiovascular system diseases and disorders, the medications prescribed by the cardiologist, etc.

Dental treatment of chronic periodontitis based on the proposed concept is a paradigm that opens new perspectives in the development of interdisciplinary and personalized dentistry, based on principles that emphasize the fundamental relationships between dental diseases, the general condition of the organism, metabolism and the internal organs that are multidirectionally involved in pathological processes.

\section{Conclusions}

The Index Myocardium (ECG dispersion mapping) positively correlates with the severity and duration of the periodontal disease, patient's age and the systolic blood pressure level. Around $85 \%$ of patients with chronic periodontitis had preclinical ischemic signs, revealed by changes of the ECG dispersion mapping of myocardial tissues.

The subsequent dental treatment for patients with high initial values of the Index Myocardium (IM > 50) or with an aggravation of the preclinical ischemic signs during dental procedures will require the surveillance of the cardiologist.

The periodontitis-target organ (heart) concept represents a cumulative synthesis of the available data from literature and our investigations, and it may prove as a step towards an integrative medicine approach in periodontology.

\section{Author Contributions}

VF: Idea, experimental design, performed investigations, data analysis, manuscript writing. VL: Idea, experimental design, data gathering, analysis and interpretation, manuscript writing. SC: Data analysis, manuscript proofreading. GB: Data analysis, manuscript proofreading.

\section{Acknowledgments}

The authors have no conflicts of interest. This research did not receive any specific grant from funding agencies in the public, commercial, or nonprofit sectors. The authors declare that the research was conducted in the absence of any commercial or financial relationship that could be construed as a potential conflict of interest. 


\section{References}

1. Eke PI, Dye BA, Wei L, et al. Prevalence of periodontitis in adults in the United States: 2009 and 2010. J Dent Res. 2012;91(10):914-920. [Full text links] [CrossRef] [PubMed] Google Scholar Scopus

2. Page RC, Eke PI. Case definitions for use in populationbased surveillance of periodontitis. J Periodontol. 2007;78(7 Suppl):1387-1399.

[Full text links] [CrossRef] [PubMed] Google Scholar Scopus

3. Lockhart PB, Bolger AF, Papapanou PN, et al. Periodontal disease and atherosclerotic vascular disease: does the evidence support an independent association? Circulation. 2012;125(20):2520-2544.

[Full text links] [CrossRef] [PubMed] Google Scholar

4. Mârțu S. [Periodontal medicine - a deep medical concept in dentistry]. [Article in Romanian]. Rom J Periodont. 2010;1(1):7-16.

5. Hajishengallis G. Periodontitis: from microbial immune subversion to systemic inflammation. Nat Rev Immunol. 2015;15(1):30-44.

[Full text links] [CrossRef] [PubMed] Google Scholar Scopus

6. Chi H, Messas E, Levine RA, et al. Interleukin-1 receptor signaling mediates atherosclerosis associated with bacterial exposure and/ or a high-fat diet in a murine apolipoprotein $\mathrm{E}$ heterozygote model: pharmacotherapeutic implications. Circulation. 2004;110(12):1678-1685.

[Full text links] [CrossRef] [PubMed] Google Scholar

7. Ryabykina GV, Sula AS. [Applying CardioVisor -06 in screening examination. Method of dispersion plotting] [in Russian]. Moscow, RU: Ministry of Public Health of Russia; 2004.

8. Fala V. [BioR - the basis for optimizing tissue regeneration processes] [in Romanian]. Chişinău, MD: HM Design; 2014.

9. Saini R, Saini S, Saini SR. Periodontal diseases: a risk factor to cardiovascular disease. Ann Card Anaesth. 2010;13(2):159-161.

[Full text links] [CrossRef] [PubMed] Google Scholar Scopus

10. Tonetti MS, Van Dyke TE; working group 1 of the joint EFP/ AAP workshop. Periodontitis and atherosclerotic cardiovascular disease: consensus report of the Joint EFP/AAP Workshop on Periodontitis and Systemic Diseases. J Periodontol. 2013;84(4 Suppl):S24-S29.

[Full text links] [CrossRef] [PubMed] Google Scholar
11. Marsh PD, Martin MV. Oral microbiology. 5th Edition. Salisbury, UK: Elsevier Health Sciences; 2009.

12. Abdulazim $A$, Stienen $M N$, Sadr-Eshkevari $P$, et al Trigeminocardiac reflex in neurosurgery - current knowledge and prospects. 2015.

Google Scholar

13. Arakeri G, Arali V. A new hypothesis of cause of syncope: trigeminocardiac reflex during extraction of teeth. Med Hypotheses. 2009;74(2):248-251.

[Full text links] [CrossRef] [PubMed] Google Scholar Scopus

14. Elter JR, Hinderliter AL, Offenbacher $S$, et al. The effects of periodontal therapy on vascular endothelial function: a pilot trial. Am Heart J. 2006;151(1):47.

[Full text links] [CrossRef] [PubMed] Google Scholar Scopus

15. Pradhan AD, Ridker PM. Do atherosclerosis and type 2 diabetes share a common inflammatory basis? Eur Heart J. 2002;23(11):831-834.

[Full text links] [CrossRef] [PubMed] Google Scholar

16. MacDougall R. NIH Human Microbiome Project defines normal bacterial makeup of the body. 2012. http://www.nih.gov/news/ health/jun2012/nhgri-13.htm

[PubMed]

17. Michalowicz BS, Hodges JS, DiAngelis AJ, et al. Treatment of periodontal disease and the risk of preterm birth. $N$ Engl J Med. 2006;355(18):1885-1894.

[Full text links] [CrossRef] [PubMed] Google Scholar Scopus

18. Schaefer AS, Richter GM, Groessner-Schreiber B, et al. Identification of a shared genetic susceptibility locus for coronary heart disease and periodontitis. PLoS Genet. 2009;5(2):e1000378.

[Full text links] [Free PMC Article] [CrossRef] [PubMed] Google Scholar Scopus

19. Lăcustă V. [Direct transcranial stimulation with direct current] [in Romanian]. Chişinău, MD: Elena VI; 2011.

20. Puhar I, Kapudija A, Kasaj A, et al. Efficacy of electrical neuromuscular stimulation in the treatment of chronic periodontitis. J Periodontal Implant Sci. 2011;41(30):117-122.

[CrossRef] [PubMed] Google Scholar Scopus

Valeriu FALA
DMD, PhD, MSc, Associate Professor,
Head Department of Therapeutic Dentistry
Faculty of Dentistry“Nicolae Testemițanu” State University of Medicine and Pharmacy
Chișinău, Republic of Moldova

Author of more than 55 inventions in the field of dentistry. Participant at national and international invention fairs (Moldova, Romania, Ukraine, USA, Belgium, Switzerland, China, Poland, Germany, Czech Republic, Spain), awarded with 61 medals, including 26 gold, 19 silver and 11 bronze medals and others.

In 2010, his practice received WIPO (World Intellectual Property Organization) Award for Innovative Enterprises. In 2018, he has received the WIPO Award for Innovational Activity. Author of more than 92 scientific articles in national and international journals. Author of clinical guidebooks and monographs. Double champion $(2001,2004)$ of the International Contest for Restorative Dentistry “Prisma Championship” (Poltava, Ukraine). 1st place at the International Dental Contest of Clinical Cases, Moscow, Russia, 2004. Vice-president of Association of Stomatologists of Republic of Moldova (ASRM). 


\section{Ouestions}

1. How many individuals are forecasted to have periodontitis in US population in 2030 ?

Da. One in ten;

ab. One in two;

ac. One in three;

ad. One in five.

2. What does a high value of MI (myocardial index) mean?

$\square$ a. A high value means less myocardial ischemic disorders;

ab. A low value means higher myocardial ischemic disorders;

D. A high value means higher myocardial ischemic disorders;

$\square$ d. A low value means less myocardial ischemic disorders.

\section{What is the sensitivity of the ECG dispersion mapping?}

a. 78\%;

ab. $80 \%$;

口c. $99 \%$;

ad. $26 \%$.

4. How big is the risk for developing severe periodontal disease in individuals with a positive genotype (detected on periodontitis-susceptibility test)?

$\square$ a. 2 times higher;

ab. 6,8 times higher;

ac. 4 times higher;

$\square d$. It is the same as in individuals with negative genotype.

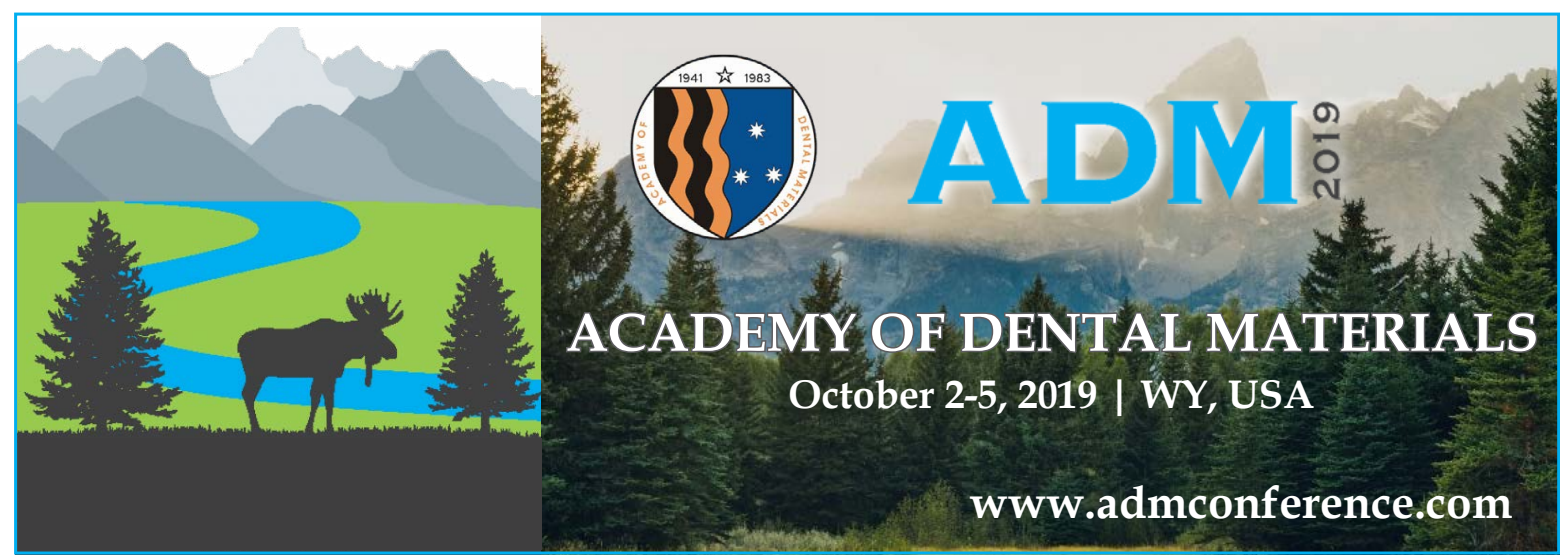

